Revista de Psicología de la PUCP. Vol. XXI, 2, 2003

\title{
Indicadores de homosexualidad en el Dibujo de la Figura Humana: comparación entre homosexuales y heterosexuales ${ }^{1}$
}

\author{
Percy Fernández Dávila² y Daniel Ávila ${ }^{3}$ \\ Pontificia Universidad Católica del Perú
}

\begin{abstract}
El estudio revisa los indicadores gráficos (IG) sobre homosexualidad en el Dibujo de la Figura Humana descritos en diversos textos sobre pruebas proyectivas. Participaron 36 hombres homosexuales y 30 hombres heterosexuales de clase media (18-50 años), quienes realizaron tres dibujos (hombre, mujer y sí mismo). Se analizaron los IG resultantes del estudio, la información de la literatura y lo que señalan un grupo de psicólogos sobre los IG que describen homosexualidad. Los resultados revelan que no existen mayores diferencias entre los IG de los dibujos de los hombres homosexuales y de los heterosexuales; sólo se encontraron tres indicadores característicos en los hombres homosexuales (Boca Arco de Cupido en la Figura Femenina, Ojos Grandes/Remarcados en la Figura Sí Mismo y Figura Semidesnuda en las figuras Femenina y Sí Mismo) y un indicador en los hombres heterosexuales (Ojos Vacíos en la Figura Masculina). Casi todos los IG que señala la literatura no resultan válidos para describir las homosexualidades y los clínicos manejan concepciones estereotipadas sobre la homosexualidad. Palabras clave: Dibujo de la Figura Humana, pruebas psicológicas, homosexualidad, identidad sexual, clase media.
\end{abstract}

Indicators of homosexuality in the Human Figure Test: comparison between homosexual and heterosexual men

This study reviews graphic indicators (GI) of homosexuality in the Human Figure Drawing Test (HFDT) which have been described in various texts on projective tests. 36 homosexual and 30 heterosexual men were enrolled, all middle-class and aged 18-50. Each participant drew three figures (man, woman and themselves). Information from the literature, the GIs from the study, and the expertise of a group of psychologists concerning which GIs describe homosexuality in HFDTs were analyzed. Results show that large differences do not exist between GIs of drawings by homosexual men and heterosexual men. However, three characteristic indicators were found in the drawings of homosexual men (Semi-Nude Female and Self Figures, Female Figure with Cupid Arch Mouth, and Self Figure with Large/ Emphasized Eyes) and one indicator in those by heterosexual males (Male Figure with Empty Eyes). Almost all of the GIs found in the literature fail to describe homosexuality and that clinicians continue to be influenced by stereotypic conceptions of homosexuality.

Key words: Human Figure Drawing Test, psychological tests, homosexuality, sexual identity, middle class.

Trabajo presentado en el IV Congreso Iberoamericano de Evaluación Psicológica, Lima, Perú, 2003. 

Este estudio nace a partir de la observación que hace uno de los autores en su experiencia de trabajo clínico, cuando al realizar evaluaciones con el Test del Dibujo de la Figura Humana (TDFH) a ciertos hombres con orientación homosexual (homosexuales masculinos, principalmente) advierte que no aparecían muchos de los indicadores gráficos que describe la literatura sobre homosexualidad. Esto originó la hipotesis de que muchos de los indicadores gráficos descritos para homosexualidad en el TDFH fueron determinados a partir de lo encontrado en cierta población homosexual (v.g. homosexuales travestíes o femeninos), generalizándose equivocadamente sin tomar en consideración otras formas de ser homosexual (v.g. homosexuales masculinos).

Otra motivación para realizar este estudio parte de una crítica a la visión heterosexista y normalizadora que tiene aún la psicología con respecto a la sexualidad y la identidad sexual cuando se utilizan tests psicológicos como referentes para describir la conducta sexual.

\section{Antecedentes}

El TDFH es un test proyectivo que evalúa aspectos de la personalidad y ajuste emocional con relación al autoconcepto y la imagen corporal.

2 Psicólogo clínico egresado de la Pontificia Universidad Católica del Perú. Actualmente trabaja en la Universidad Peruana Cayetano Heredia, donde se dedica a investigación, docencia y prevención en las áreas de sexualidad, género, salud sexual y SIDA, focalizando su trabajo con grupos vulnerables y población marginal. Correo electrónico: percyfer@terra.com.pe.

3 Estudiante del último año de la especialidad de Psicología Clínica de la Pontificia Universidad Católica del Perú, interesándose por los campos de la estadística y la investigación. Correo electrónico: danyavila82@ hotmail.com. 
Este test es utilizado en el Perú, principalmente, con fines diagnóstico y predictivo (Niño de Guzmán, Fernández Dávila, Lira \& Warthon, 2000).

El TDFH y las pruebas proyectivas gráficas en general son la segunda técnica de evaluación psicológica más utilizada en el Perú (Ráez, 1999) y en los EEUU (Watkins, Campbell, Nieberding \& Hallmark, 1995) después del Psicodiagnóstico de Rorschach. Sin embargo, la escasa investigación empírica que existe en nuestro medio hace que se utilicen datos de otras realidades socioculturales, que pueden ser peligrosos al momento de determinar una valoración psicológica.

Con respecto a la homosexualidad, ésta se entiende para los propósitos de este estudio como una orientación o atracción sexual, es decir, el deseo físico, erótico, así como la disposición imaginativa y afectiva hacia alguien del mismo sexo (Kornblit, Pecheny \& Vujosevich, 1998). En este sentido, se la considera como una cualidad estructural. Sin embargo, se debe hacer una distinción con el concepto de identidad homosexual, el cual vendría a ser la coincidencia de deseos, sentimientos, actos y conciencia, que culminan en la aceptación de uno mismo como homosexual, en un proceso de autodefinición (Castañeda, 1999). Es decir, alguien puede ser homosexual (latente o manifiesto, egodistónico o egosintónico) y no tener una identidad homosexual.

Tradicionalmente, los hombres homosexuales son conceptualizados desde los más diversos ámbitos: la religión, las ciencias, el Estado y la política (Jiménez, 1998). Las ideas sobre la homosexualidad se estructuran sobre la base de diversas imágenes y estereotipos que distorsionan a la persona homosexual. En la mayoría de los casos, se piensa que los hombres homosexuales son afeminados o pretenden ser mujeres. Sin embargo, no existe una sola forma de ser homosexual, existen formas de ser homosexual (homosexualidades), que pueden ir desde un homosexual travestí hasta un homosexual que se siente "supermacho". 
Los indicadores gráficos en el TDFH que señala la literatura y que describen "homosexualidad en general", sin considerar la diversidad señalada anteriormente, son diversos. Así, los que aparecen en la mayor parte de los textos especializados (Grassano, 2000; Levy, 1978; Machover, 1949; Portuondo, 1983) con mayor frecuencia son:

- Primer dibujo es del sexo opuesto

- Figura masculina/femenina con caracteres secundarios femeninos/ masculinos

- Acentuación/sombreado del cabello

- Pestañas

- Labios gruesos en forma de "arco de cupido"

- Tronco redondeado

- Cintura estrecha

- Cadera ancha en la figura masculina

- Corbata

- Tacos en los zapatos masculinos

- Pliegues o líneas en la zona genital

Como se puede observar, la mayor parte de estos indicadores tienen un significado interpretativo que indica afeminamiento, inadecuación o conflictos sexuales y dudas acerca de la masculinidad o la virilidad.

\section{Objetivos}

- Determinar el conjunto de indicadores que aparecen con mayor frecuencia en los dibujos del TDFH, en el grupo de hombres homosexuales y de hombres heterosexuales.

- Determinar qué indicadores y en qué proporción tienen una ausencia importante y significativa dentro del grupo de hombres homosexuales y dentro del grupo de hombres heterosexuales. 
- Determinar las agrupaciones de indicadores (factores) que puedan aparecer y que sean estadísticamente significativas en cada grupo poblacional.

- Comparar los indicadores que resulten significativos en el grupo de homosexuales con los que refiere la literatura psicológica y con los que determinan un grupo de psicólogos que utilizan el TDFH en su practica clínica para describir homosexualidad.

\section{Metodología}

\section{Diseño}

Este es un estudio premilinar de uno más amplio. La investigación se inscribe dentro de los estudios descriptivo-comparativos, de corte transversal (Hernández, Fernández \& Baptista, 1991). Se realizó una triangulación de datos (Arias, 1999) por la que se buscó una comparación cruzada de los indicadores gráficos que provengan de los resultados de este estudio con la información proveniente de la literatura y lo que señalan un grupo de psicólogos sobre los indicadores gráficos que describen homosexualidad en el TDFH.

\section{Participantes}

Los participantes fueron seleccionados a partir de un muestreo no probabilístico, de tipo accidental, a través de la técnica de la "bola de nieve". Se reclutaron 36 hombres homosexuales masculinos ${ }^{4}$, con identidad homosexual asumida, y 30 hombres heterosexuales de la población general de clase media. Debido a la dificultad de definir claramente la clase media en el Perú (Portocarrero, 1998), se consideró

4 Se escogió trabajar con hombres homosexuales masculinos de clase media porque en este sector ellos representan un tipo particular de ser homosexual, valoran y defienden su identidad masculina por encima de ser homosexual (Cáceres, Salazar, Rosasco \& Fernández Dávila, 2002). 
más conveniente equiparar el concepto con el de nivel socioeconómico, definido a partir de las siguientes características: grado de instrucción, ocupación y lugar de residencia.

Los participantes de ambos grupos presentan similares características sociodemográficas:

- Las edades fluctúan entre los 18 y 50 años (medias: 34 años, hombres homosexuales, y 32 años, hombres heterosexuales).

- Todos los participantes cuentan con nivel de educación superior (técnica o universitaria).

- La mayoría son profesionales, empresarios, trabajadores independientes y empleados.

- Más del $80 \%$ son nacidos en Lima, el resto son provincianos y algunos extranjeros residentes de larga data en Lima.

- La mayoría vive en distritos residenciales considerados de clase media (INEI, 1998).

Características de los jueces: Los criterios de inclusión para considerar como expertos a los profesionales que utilizan el TDFH fueron: psicólogos con ejercicio clínico y experiencia mayor a cuatro años en el uso del TDFH. Así, se obtuvo el siguiente perfil:

- Nueve psicólogos, de ambos sexos, con ejercicio clínico, todos con alguna experiencia docente en la enseñanza de pruebas psicológicas.

- $\quad$ Edad promedio de 32 años.

- Ocho años en promedio de tiempo de práctica profesional y, también, ocho años de experiencia en el uso de tests proyectivos gráficos y, sobretodo, del TDFH. 
Percy Fernández Dávila y Daniel Ávila

\section{Instrumento}

Se utilizó el TDFH (Machover, 1949) en las versiones de Hombre, Mujer y Sí Mismo. Se incluyó el dibujo del sí mismo porque pareció interesante recoger la autopercepción de los hombres homosexuales, es decir, cómo se ven ellos mismos.

Se elaboró un sistema objetivo de calificación adaptado de otros sistemas similares de evaluación del TDFH (Marsh, Linberg \& Smeltzer, 1991; Naglieri, McNeish \& Bardos, 1991; Niño de Guzmán et al., 2000), lo que le otorga una validez de constructo. El sistema está compuesto por 113 indicadores gráficos definidos operacionalmente (ver Anexo). A través de él, se evalúa la presencia de los indicadores, tanto en los aspectos formales (ubicación, tamaño, tipo de línea, completud, sombreado, etc.) como en los de contenido (detalles, accesorios, otros objetos, etc.) de los dibujos. Se obtuvo una confiabilidad intercalificadores de 0.95 , un indicador que resulta alto y que demuestra su consistencia cuando diferentes evaluadores califican un mismo protocolo.

\section{Procedimiento}

El contacto con los participantes, en ambos grupos de estudio, se hizo a partir de la referencia que hacía un participante a otros similares (conocido como la "bola de nieve" o "radio bemba"), previa consulta a éstos. Luego, se realizó la aplicación de un consentimiento informado oral, donde se describía a los participantes los objetivos y fines del estudio, garantizando el anonimato y la confidencialidad. El tiempo de administración promedio del TDFH fue de 10 minutos. La aplicación incluía que los participantes narraran una historia para cada figura, excepto para la Figura de Sí Mismo, pero que por razones de extensión, tiempo y complejidad no fueron incluidas en el análisis. Paralelamente al recojo de datos con la población objetivo, se solicitó a nueve jueces (psicólogos), con experiencia en el uso de técnicas 
proyectivas gráficas, su colaboración en evaluar los ítems propuestos en el sistema de calificación del TDFH, para determinar su criterio sobre cuáles indicadores describen homosexualidad. Finalmente, se procedió a la calificación de las figuras a partir del sistema propuesto.

Acabada la calificación, se procedió a ingresar los datos en el software estadístico SPSS. Nos interesó realizar un análisis descriptivo de los datos para obtener distribución de frecuencias y proporciones. También se hizo uso de la estadística inferencial para el contraste de hipótesis a través de un análisis no paramétrico, utilizando la Prueba $\mathrm{Z}$ sobre la diferencia de proporciones para muestras independientes.

\section{Resultados}

\section{Análisis descriptivo de los jueces}

En el Cuadro 1 se describen los indicadores gráficos que fueron señalados con mayor frecuencia por los jueces como signos que describen homosexualidad. Un acuerdo total de $100 \%$ fue otorgado a Impresión Femenina/Masculina de la figura que no corresponde a su sexo, seguido por Figura con Caracteres Sexuales Secundarios Acentuados (89\%), Figura Indiferenciada Sexualmente y Bragueta/ Pliegues (ambos con 78\%), Pelvis Sombreada/Borroneada y Pestañas (ambos con 67\%); y, Manos Ocultas, Borraduras y Pelo en Otras Partes del Cuerpo, todos ellos con un $56 \%$ de coincidencia.

\section{Análisis descriptivo de los participantes}

A continuación se presenta el conjunto de indicadores que aparecen con mayor frecuencia en las tres figuras. Los datos son presentados como proporciones. 


\section{Cuadro 1}

Indicadores señalados para desribir homosexualidad según jueces*

\begin{tabular}{|l|c|}
\hline \multicolumn{1}{|c|}{ Indicadores } & Porcentaje \\
\hline Impresión femenina/masculina de la figura que no & \\
corresponde a su sexo & $100 \%$ \\
Figura con caracteres sexuales secundarios acentuados & $89 \%$ \\
Figura indiferenciada sexualmente & $78 \%$ \\
Bragueta o pliegues & $78 \%$ \\
Pelvis sombreada o borroneada & $67 \%$ \\
Pestañas & $67 \%$ \\
Manos ocultas & $56 \%$ \\
Borraduras & $56 \%$ \\
Pelo en otras partes del cuerpo & $56 \%$ \\
\hline
\end{tabular}

* Indicadores con acuerdo mayor a 50\%.

En el Cuadro 2 se comparan los indicadores que aparecieron con mayor ocurrencia en la Figura Hombre tanto del grupo homosexual como en el grupo heterosexual. Así, Línea Base/Piso (0.58 vs. 0.63), Figura Muy Grande ( 0.31 vs. 0.27$)$, Braguetas o Pliegues ( 0.25 vs. 0.30 ), Línea Entrecortada/Discontinua (0.28 vs. 0.27), Cuello Muy Largo (0.17 vs. 0.10$)$ y Ojos Vacíos (0.06 vs. 0.22$)$ fueron los que aparecen con mayor frecuencia.

En el Cuadro 3, para la Figura Mujer, aparecen con mayor frecuencia: Línea de Base/Piso (0.47 vs. 0.57), Pelo Acentuado (0.31 vs. 0.17), Línea Entrecortada/Discontinua (0.28 vs. 0.30), Tacos (0.28 vs. 0.20 ), Boca Tipo Arco de Cupido (0.28 vs. 0.00), Ojos Grandes/ Remarcados ( 0.22 vs. 0.10$)$ y Figura Semidesnuda (0.14 vs. 0.00$)$. 
Indicadores de homosexualidad en el Dibujo de la Figura Humana

Cuadro 2

Figura Hombre*

\begin{tabular}{|l|c|c|}
\hline \multicolumn{1}{|c|}{ Indicadores } & Homosexuales & Heterosexuales \\
\hline Figura muy grande & 0.31 & 0.27 \\
Ubicación superior & 0.17 & 0.10 \\
Línea de base/piso & 0.58 & 0.63 \\
Línea entrecortada/discontinua & 0.28 & 0.27 \\
Pelo acentuado & 0.22 & 0.17 \\
Cuello muy largo & 0.17 & 0.10 \\
Ojos mirando hacia un lado & 0.22 & 0.17 \\
Braguetas o pliegues & 0.25 & 0.30 \\
Ojos vacíos & 0.06 & 0.22 \\
\hline
\end{tabular}

* Indicadores con proporción mayor a 0,17

Cuadro 3

Figura Mujer*

\begin{tabular}{|l|c|c|}
\hline \multicolumn{1}{|c|}{ Indicadores } & Homosexuales & Heterosexuales \\
\hline Figura muy grande & 0.28 & 0.30 \\
Figura muy pequeña & 0.17 & 0.17 \\
Línea de base/piso & 0.47 & 0.57 \\
Línea entrecortada/discontinua & 0.28 & 0.30 \\
Pelo acentuado & 0.31 & 0.17 \\
Ojos mirando hacia un lado & 0.22 & 0.23 \\
Tacos & 0.28 & 0.20 \\
Ojos grandes/remarcados & 0.22 & 0.10 \\
Boca tipo "arco de cupido" & 0.28 & 0.00 \\
Cuello muy largo & 0.11 & 0.17 \\
Ojos vacíos & 0.06 & 0.17 \\
Ubicación superior & 0.08 & 0.17 \\
Figura semidesnuda & 0.14 & 0.00 \\
\hline
\end{tabular}

* Indicadores con proporción mayor a 0,14 
En el Cuadro 4, para la Figura Sí Mismo, aparecen con mayor frecuencia: Línea de Base/Piso (0.47 vs. 0.60), Figura Muy Grande ( 0.44 vs. 0.30 ), Línea Entrecortada/Discontinua ( 0.36 vs. 0.37 ), Ojos grandes/Remarcados (0.22 vs. 0.10), Figura Semidesnuda (0.11 vs. $0.00)$ y Botones y/o Bolsillos en Exceso (0.19 vs. 0.30).

\section{Cuadro 4}

Figura Si Mismo*

\begin{tabular}{|l|c|c|}
\hline \multicolumn{1}{|c|}{ Indicadores } & Homosexuales & Heterosexuales \\
\hline Figura muy grande & 0.44 & 0.30 \\
Línea de base/piso & 0.47 & 0.60 \\
Línea entrecortada/discontinua & 0.36 & 0.37 \\
Bragueta o pliegues & 0.25 & 0.27 \\
Botones y/o bolsillos en exceso & 0.19 & 0.30 \\
Ojos grandes/remarcados & 0.31 & 0.10 \\
Brazos pegados al cuerpo & 0.19 & 0.07 \\
Figura semidesnuda & 0.11 & 0.00 \\
\hline
\end{tabular}

* Indicadores con proporción mayor a 0,11

\section{Análisis inferencial}

En los datos que se presentan en el Cuadro 5, sólo se han incluido los indicadores que resultaron con un valor estadísticamente significativo. Para esto se utilizó el análisis no paramétrico llamado Prueba $Z$ sobre la diferencia de proporciones para muestras independientes.

Para la Figura Hombre resultó estadísticamente significativo y con una diferencia grande a favor del grupo heterosexual el indicador Ojos Vacíos $\left(\mathrm{Z}>\mathrm{Z}_{0.975}\right)$. Para la Figura Mujer resultaron diferentes y significativos a favor del grupo homosexual los indicadores Boca Tipo Arco de Cupido y Figura Semidesnuda (ambos con $\mathrm{Z}>\mathrm{Z}_{0.975}$ ). Para la Figura Sí Mismo resultó diferente y significativo, a favor del grupo homosexual, el indicador Ojos Grandes Remarcados $\left(\mathrm{Z}>\mathrm{Z}_{0.975}\right)$ y resultó con una presencia importante y significativa, sólo en el grupo homosexual, el indicador Figura Semidesnuda $\left(\mathrm{Z}>\mathrm{Z}_{0.95}\right)$. 
Indicadores de homosexualidad en el Dibujo de la Figura Humana

\section{Cuadro 5}

Contraste sobre la diferencia de proporciones para muestras independientes

\begin{tabular}{|c|c|c|c|c|c|c|c|c|c|}
\hline Figura & Indicador & $\begin{array}{c}\text { Homose } \\
\text { P1 }\end{array}$ & $\begin{array}{l}\text { Heterose } \\
\text { P2 }\end{array}$ & $\begin{array}{c}\text { Hipótesis } \\
\mathbf{H}_{1}\end{array}$ & $\mathbf{Z}$ & $\begin{array}{c}Z \\
(0.95)\end{array}$ & \begin{tabular}{|c|}
$Z$ \\
$(0.975)$
\end{tabular} & $\begin{array}{l}\text { H de } \\
\text { Cohen }\end{array}$ & Tamaño \\
\hline $\begin{array}{l}\text { Figura } \\
\text { Hombre }\end{array}$ & Ojos vacíos & 0.06 & 0.22 & $\mathrm{P} 1=\mathrm{P} 2$ & 1.975 & & & 0.5003 & \\
\hline \multirow{2}{*}{$\begin{array}{l}\text { Figura } \\
\text { Mujer }\end{array}$} & $\begin{array}{l}\text { Boca tipo } \\
\text { arco de } \\
\text { cupido }\end{array}$ & 0.28 & 0.00 & $\mathrm{P} 1=\mathrm{P} 2$ & 3.149 & & & 1.1152 & \\
\hline & $\begin{array}{l}\text { Figura } \\
\text { semidesnuda }\end{array}$ & 0.14 & 0.00 & $\mathrm{P} 1=\mathrm{P} 2$ & 2.132 & 1.645 & 1.96 & 0.7670 & Grande \\
\hline \multirow{2}{*}{$\begin{array}{c}\text { Figura } \\
\text { Sí Mismo }\end{array}$} & $\begin{array}{l}\text { Ojos grandes } \\
\text { remarcados }\end{array}$ & 0.31 & 0.10 & $\mathrm{P} 1 \cdot \mathrm{P} 2$ & 2.069 & & & 0.5375 & \\
\hline & $\begin{array}{l}\text { Figura } \\
\text { semidesnuda }\end{array}$ & 0.11 & 0.00 & $\mathrm{P} 1>\mathrm{P} 2$ & 1.874 & & & 0.6761 & \\
\hline
\end{tabular}

Debido a que los indicadores resultantes del análisis inferencial para las tres figuras son pocos, no se pudo realizar un análisis factorial para determinar agrupaciones estadísticamente relevantes.

\section{Discusión}

Debemos mencionar que ningún indicador que pueda orientarnos a una interpretación que ayude a describir al grupo homosexual de esta muestra tiene una presencia elevada, sin embargo, sus ocurrencias nos pueden ayudar a describir tendencias u orientaciones.

Como se ha podido observar en las tres figuras (ver Cuadros 2, 3 y 4), ambos grupos puntúan de similar manera en casi todos los indicadores que se muestran. Los indicadores Figura Muy Alta, Línea Base, Línea Entrecortada/Discontinua y Bragueta (sólo en la Figura Hombre y la Figura Sí Mismo) resaltan tanto en los hombres homosexuales como en los heterosexuales, por lo que estos indicadores 
se deben considerar como características prevalentes de la población general. Sin embargo, consideramos que el indicador Línea Base es un indicador que ya no resulta válido en el TDFH por estar "contaminado"; ha pasado a formar parte del ícono popular que no se debe dejar de dibujar cuando la gente es evaluada con este test. Con respecto al indicador Figura Muy Alta, para dar una valoración interpretativa, más importante que el tamaño absoluto, es la impresión que transmite la relación entre la figura y el espacio que la circunda, lo que amerita, cualitativamente, una mirada más profunda a los dibujos. Por otro lado, llama la atención el dibujo de la Bragueta (interpretado como marcador de preocupación o conflicto sexual) que aparece ligeramente con mayor frecuencia en los hombres heterosexuales, contrariamente a lo que se hubiese pensado.

Por otra parte, se resaltan también algunas diferencias. En la Figura Hombre (ver Cuadro 3), el Cuello Muy Largo aparece en mayor grado en los hombres homosexuales, lo cual puede ser interpretado, tal vez, como control o represión del deseo en algunos de los hombres homosexuales. Los Ojos Vacíos tienen una diferencia marcadamente superior a favor de los hombres heterosexuales lo que según el contraste inferencial (ver Cuadro 5) resulta estadísticamente significativo para este grupo. Este indicador, en un porcentaje de hombres heterosexuales, se puede interpretar como un signo egocéntrico que puede guardar relación, quizá, con un estilo más individualista, centrado en sí mismo, de manejarse y ver el mundo, y que puede ser propio de personas con éxito personal y social.

En la Figura Mujer (ver Cuadro 4) aparecen el Pelo Acentuado, los Tacos y la Boca Tipo Arco de Cupido con mayor ocurrencia en los hombres homosexuales frente a los hombres heterosexuales. Estos indicadores son mencionados por la literatura como los más frecuentes cuando se describe homosexualidad. Sin embargo, su frecuencia y significancia no resulta importante, salvo el indicador Boca Tipo Arco de Cupido, el cual es estadísticamente significativo para el grupo de 
hombres homosexuales (ver Cuadro 5). Este indicador se puede relacionar a la sensualidad y a la expresividad sexual. Asimismo, llama la atención que en ambos grupos aparezca el indicador Figura Pequeña en este dibujo, lo que significaría que para algunos hombres de la muestra completa, la mujer es percibida como inferior o con poco valor.

En la Figura Sí Mismo (ver Cuadro 5) aparecen como más frecuente en los hombres heterosexuales los Botones y Bolsillos, lo cual está asociado con dependencia afectiva. Los Ojos Grandes/Remarcados aparecen con frecuencia alta y estadísticamente significativa en los hombres homosexuales. Creemos que este indicador, en un grupo de hombres homosexuales, tiene que ver con un estado de alerta o hipervigilancia, entendible en esta población por el grado de cuidado, discreción y reserva con la que manejan su identidad, por miedo o temor al rechazo o estigma social. Sin embargo, no consideramos que este signo sea un indicador propio dentro del grupo homosexual sino más bien de cualquier persona o grupo que esté sometido a un grado de exigencia o cuidado de su conducta con respecto a su entorno.

El indicador Figura Semidesnuda, a pesar de tener una ocurrencia relativamente baja, aparece estadísticamente significativo en el grupo de hombres homosexuales tanto en la Figura Mujer como en la Figura Sí Mismo (ver Cuadro 5). Este indicador puede estar asociado con una actitud contestataria, de ir en contra de lo que socialmente se impone. Esto es entendible puesto que dentro del grupo homosexual pueden haber algúnos hombres que asumen su condición con ánimos de enfrentamiento o con un manejo alternativo hacia lo que puede ser socialmente impuesto.

Por último, los indicadores gráficos que señalan los jueces para describir homosexualidad ${ }^{5}$ (ver Cuadro 1) están orientados a enfatizar

5 Solamente dos psicólogos solicitaron clarificación con respecto a la concepción de la homosexualidad. 
inversión sexual, confusión con respecto a la identidad sexual, afeminamiento, conflictos sexuales, sentimientos de insuficiencia masculina y ansiedad. La mayoría de estos indicadores guardan un correlato directo con los indicadores gráficos que propone la literatura para describir homosexualidad, por lo que pensamos que la mayoría de psicólogos hacen valoraciones sin cuestionar o relativizar la "prescripción" que señalan los textos. Y quizá no la cuestionan o relativizan porque mantienen imágenes arquetípicas o características estereotipadas sobre la forma de ser homosexual.

\section{Conclusiones y recomendaciones}

- No existen mayores diferencias entre los indicadores gráficos de los dibujos del TDFH de los hombres homosexuales comparados con los de los heterosexuales.

- Sin embargo, se encontraron tres indicadores que resultaron característicos en los hombres homosexuales (Boca Tipo Arco de Cupido y Figura Semidesnuda en la Figura Mujer; Ojos Grandes/ Remarcados y Figura Semidesnuda en la Figura Sí Mismo), y un indicador en los hombres heterosexuales (Ojos Vacíos en la Figura Hombre).

- El sistema de calificación objetivo resulta consistente para determinar y describir indicadores gráficos en el TDFH.

- Casi todos los indicadores sobre homosexualidad que señala la literatura no resultan válidos para las diversas formas de ser homosexual (salvo el indicador Boca Tipo Arco de Cupido en la Figura Mujer). Estos indicadores se orientan a conceptualizar la homosexualidad como un problema de la identificación sexual y de género.

- A partir de los criterios que utilizan los profesionales para describir homosexualidad en el TDFH, se puede deducir que concepciones e imágenes estereotipadas y prejuiciosas sobre la homosexualidad (asociadas a conflictos con la sexualidad y la 
identidad de género y a identificar lo homosexual como femenino), así como una confusión conceptual entre identidad sexual e identidad de género, se mantienen entre los profesionales que utilizan el TDFH.

- Ampliar el tamaño de la muestra para verificar y/o potenciar estos resultados preliminares.

- Incluir otros grupos, con otras formas de ser homosexual (v.g. homosexuales travestíes $\mathrm{u}$ afeminados), y compararlos con la muestra actual para determinar diferencias.

- Seguir trabajando con el sistema de calificación propuesto en diferentes poblaciones para poder optimizar la capacidad discriminativa del test.

\section{Referencias}

Arias, M. (1999). La triangulación metodológica: sus principios, alcances y limitaciones. Investigación y Educación en Enfermería, 18. Recuperado de http://tone.udea.edu.co/ revista/mar2000/ Triangulacion.html

Cáceres, C., Salazar, X., Rosasco, A.M. \& Fernández Dávila, P. (2002). Ser hombre en el Perú de hoy. Una mirada a la infidelidad, la violencia y la homofobia desde la salud sexual. Lima: REDESS Jóvenes.

Castañeda, M. (1999). La experiencia homosexual. Para comprender la homosexualidad desde dentro y desde fuera. México: Paidós.

Grassano, E. (2000). Indicadores psicopatológicos en técnicas proyectivas. Buenos Aires: Nueva Visión.

Hernández, R., Fernández, C. \& Baptista, P. (1991). Metodologia de la investigación. México: Mc Graw Hill.

Instituto Nacional de Estadística e Informática (1998). Planos estratificados de Lima Metropolitana a nivel de manzanas. Lima: INEI y Mosaic Marketing Perú. 
Jiménez, R. (1998). Ser hombre homosexual en Chile. En T. Valdés \& J. Olavarría (Eds.), Masculinidades y equidad de género en América Latina. Santiago de Chile: FLACSO.

Kornblit, A. L., Pecheny, M. \& Vujosevich, J. (1998). Gays y lesbianas. Formación de la identidad y derechos humanos. Buenos Aires: La Colmena.

Levy, S. (1978). Dibujo proyectivo de la Figura Humana. En E. Hammer, Tests proyectivos gráficos. Buenos Aires: Paidós.

Machover, K. (1949). Proyección de la personalidad en el Dibujo de la Figura Humana. La Habana: Cultural.

Marsh, D., Linberg, L. M. \& Smeltzer, J. K. (1991). Human figure drawings of adjudicated and nonadjudcated adolescents. Journal of Personality Assessment, 57 (1), 77-86.

Naglieri, J., McNeish, T. \& Bardos, A. (1991). Draw a Person:

Screening procedure for emotional disturbance. Austin: Pro-Ed.

Niño de Guzmán, I., Fernández Dávila, P., Lira, T. \& Warthon, R. (2000). Validez y confiabilidad del Dibujo de la Figura Humana en adultos a través de un estudio de comparación de grupos clínicos. Lima: Dirección Académica de Investigación de la Pontificia Universidad Católica del Perú.

Portocarrero, G. (Ed.). (1998). Las clases medias: entre la pretensión y la incertidumbre. Lima: SUR.

Portuondo, J. (1983). Test proyectivo de Karen Machover: la figura humana (4ta. ed.). Madrid: Biblioteca Nueva.

Ráez, M. (1999). Situación actual de la enseñanza del Psicodiagnóstico de Rorschach y los métodos proyectivos en el Perú. Revista de Psicología de la Pontificia Universidad Católica del Perú, 17(2), 147-167.

Watkins, C.E., Campbell, V., Nieberding, R. \& Hallmark, R. (1995). Contemporary practice of psychological assessment by clinical psychologists. Professional Psychology Research and Practice, 26(1), 54-60. 


\section{Anexos}

\section{Definición de indicadores gráficos}

1. Figura muy grande: Se puntúa si la figura excede el margen del cuadro de la plantilla 1.1 (es decir, si es mayor a los $18 \mathrm{~cm}$. de alto $\mathrm{y} / \mathrm{o} 9 \mathrm{~cm}$. de ancho). Artículos de ropa tales como sombreros o zapatos son incluidos en la medición, aunque otros objetos como cartera, maletín, mochila, bastón, no.

2. Figura muy pequeña: Se puntúa si la figura no excede el margen del cuadro de la plantilla 1.2 (es decir, si es menor de $9 \mathrm{~cm}$. de alto y/o $4.5 \mathrm{~cm}$. de ancho). Artículos de ropa tales como sombreros o zapatos son incluidos en la medición, aunque otros objetos como cartera, maletín, mochila, bastón, no.

3. Ubicación superior: Se puntúa cuando cualquier parte de la figura está por encima de la línea media de la hoja y parte de la figura cae en el recuadro superior de la plantilla 2.1. Pelo y artículos de vestir tales como sombreros o zapatos son incluidos en la medición, aunque otros objetos como cartera, maletín, mochila, bastón, no.

4. Ubicación inferior: Se puntúa cuando cualquier parte de la figura está por debajo de la línea media de la hoja y parte de la figura cae en el recuadro inferior de la plantilla 2.2. Pelo y artículos de vestir tales como sombreros o zapatos son incluidos en la medición, aunque otros objetos como cartera, maletín, mochila, bastón, no.

5. Ubicación izquierda: Toda la figura se encuentra ubicada hacia el lado izquierdo del recuadro sombreado de la plantilla 3.1. Artículos de vestir tales como sombreros o zapatos son incluidos en la medición, aunque otros objetos como cartera, maletín, mochila, bastón, no.

6. Ubicación derecha: Toda la figura se encuentra ubicada hacia el lado derecho del recuadro sombreado de la plantilla 3.2. Artículos de vestir tales como sombreros o zapatos son incluidos 
en la medición, aunque otros objetos como cartera, maletín, mochila, bastón, no.

7. Figura inclinada: La figura se encuentra inclinada $15^{\circ}$ o más con respecto al eje vertical (esto, con relación a la línea horizontal y utilizando el punto medio de la cabeza para determinar la inclinación). Utilizar la plantilla 4.

8. Figura agachada: El tronco se encuentra inclinado $75^{\circ}$ o más con relación a su mismo eje.

9. Figura sentada: La figura está sentada o en posición de cuclillas.

10. Figura de perfil: Tanto la cabeza como el cuerpo se encuentran de perfil o en perspectiva de ángulo.

11. Cabeza de frente, cuerpo de perfil: La cabeza está mirando al frente y el cuerpo se encuentra de perfil, como las figuras egipcias.

12. Cuerpo de frente, cabeza de perfil: El cuerpo de la figura está de frente y la cabeza mirando hacia un lado. Si se puntúa este ítem, ya no se califica Ojos Mirando hacia un Lado (ítem 41).

13. Hoja rotada: El dibujo es realizado colocando la hoja en posición horizontal.

14. Borraduras: Intentos reiterados y visibles de corrección a través de borraduras, o borra alguna parte de la figura que ya estaba dibujada.

15. Recomienzo: El evaluado realiza un segundo dibujo después de borrar todo o pide una hoja adicional para hacer el dibujo en una cara u hoja nueva.

16. Línea de base: Se puntúa si se dibuja una línea de base (pasto o suelo). La figura puede estar parada o no sobre ella.

17. Asimetría: Las partes de la figura no guardan proporción entre ellas. Por ejemplo, una extremidad es significativamente más larga o más corta que la otra, o la mitad superior, inferior, izquierda o derecha desproporcionada con respecto a la otra.

18. Figura incompleta: Dibujo únicamente del torso y la cabeza o sólo la cabeza en representación de toda la figura. Si se califica este ítem, ya no se puntúa ni Brazos ni Piernas Omitidas (ítems 66 y 77).

19. Sobresimplificación: La figura está realizada utilizando palotes o como un monigote. Sólo se puntúa si el sujeto insiste en dibujar 
palotes/monigotes luego de habérsele pedido una figura completa. Si se codifica este ítem ya no se codifica Figura Vacía (ítem 104).

20. Falla en integración: Existen partes del cuerpo de la figura que no llegan a juntarse claramente (no se articula cabeza con tronco, extremidades con tronco, etc.).

21. Línea tenue: El trazo de la figura es débil en la mayor parte del dibujo, dando la impresión que se va a desvanecer.

22. Línea reforzada: Las líneas del dibujo han sido repasadas, quedando una línea gruesa, reforzada en la mayor parte del dibujo. Se puede notar un relieve en el reverso de la hoja.

23. Línea entrecortada o discontinua: El trazo de la línea no es continuo o se asemeja a una línea "velluda" en la mayor parte del dibujo.

24. Líneas curvas: Los trazos de las líneas son delineados con formas curvas o redondeadas.

25. Cabeza omitida: La cabeza está totalmente omitida, ausente. Cualquier intento de representación de cabeza no puede ser calificado como omisión.

26. Cabeza pequeña: La cabeza es demasiado pequeña, hallándose en desproporción con relación al cuerpo de la figura.

27. Cabeza grande: La cabeza es demasiado grande, hallándose en desproporción con relación al cuerpo de la figura.

28. Cabeza inclinada: Únicamente la cabeza se inclina con respecto al tronco en un ángulo aproximado de $15^{\circ}$ o más.

29. Pelo omitido: La cabeza de la figura femenina se encuentra sin pelo, calva. Cualquier intento de representación de pelo en la cabeza no puede ser calificado como omisión.

30. Pelo alborotado: El cabello de la figura se encuentra significativamente alborotado, desordenado. No se puntúa Pelo Acentuado (ítem 31) si este ítem es calificado.

31. Pelo acentuado: El pelo es dibujado de manera remarcada o reforzada, con trazos fuertes, de tal manera que sea una parte llamativa de la figura.

32. Pelo tipo casco: El cabello de la figura está dibujado como un gorro, enmarcado por una línea o reborde notorio. 
33. Pelo en otras partes del cuerpo: Presencia de pelo o vello en otras partes del cuerpo como barba, bigotes o pelos en el pecho, piernas o axilas.

34. Rostro sombreado: Presencia de manchas, sombreado, oscurecimiento del rostro.

35. Rostro omitido: Ninguna señal de cara, rostro vacío. Si se puntúa este ítem, ya no se puntúa ojos, nariz, ni boca omitidos (ítems 36 , $43,46)$.

36. Ojos omitidos: La figura no presenta dibujo o trazo de ojos (si dibuja puntos no se califica). Cualquier intento de representación de ojos (incluyendo un ojo) no puede ser calificado como omisión.

37. Ojos vacíos: La figura presenta la ausencia de pupilas (círculos sin rellenar). Si existe cualquier indicación de pupila no se puntúa.

38. Ojos cerrados: La figura presenta ambos ojos cerrados. Si la figura está guiñando un ojo no se puntúa.

39. Ojos grandes/remarcados: Los ojos de la figura se encuentran excesivamente delineados, remarcados o sombreados. La pupila aparece dilatada o los ojos muy grandes con relación a la cara.

40. Ojos bizcos: Ambos ojos de la figura se encuentran mirando hacia adentro o hacia afuera.

41. Ojos mirando hacia un lado: Ambos ojos de la figura se encuentran mirando hacia una misma dirección. Si se calificó la cabeza hacia un lado no se puntúa.

42. Pestañas: Las pestañas son dibujadas de manera larga, tupida o rizada.

43. Nariz omitida: La figura no presenta ningún trazo o dibujo de la nariz. Cualquier intento de representación de nariz no puede ser calificado como omisión, por ejemplo dos puntos.

44. Nariz muy remarcada: La nariz de la figura se encuentra excesivamente delineada o reforzada.

45. Nariz muy grande: La nariz de la figura es excesivamente grande o protuberante con respecto al resto de la cara.

46. Boca omitida: La figura no presenta dibujo o trazo de boca, esta ausente. Cualquier intento de representación de boca no puede ser calificado como omisión. 
47. Boca tipo línea o fruncida: La boca de la figura se encuentra dibujada de manera apretada, arrugada, como un punto o una línea recta.

48. Boca muy remarcada: La boca de la figura se encuentra excesivamente delineada, remarcada.

49. Boca cóncava: Boca como de payaso, semiabierta, en gesto receptivo.

50. Boca tipo "arco de cupido": Boca como si fuese un arco, delineando unos labios gruesos.

51. Dientes: La figura presenta los dientes visibles.

52. Orejas muy remarcadas: Las orejas de la figura se encuentran excesivamente delineadas, sombreadas, repasadas.

53. Orejas muy grandes: Las orejas de la figura son excesivamente grandes, hallándose desproporcionadas con respecto a la cabeza de la figura.

54. Cuello omitido: La figura no presenta ningún trazo o dibujo de cuello.

55. Cuello reforzado: El cuello de la figura se encuentra excesivamente delineado, reforzado.

56. Cuello muy largo: El largo del cuello es excesivo con relación a la figura.

57. Tronco omitido: El tronco de la figura se halla ausente y los brazos y/o piernas salen del cuello o la cabeza. Si se puntúa este ítem, ya no se califica Brazos Omitidos (ítem 66).

58. Tronco redondeado: El tronco está delineado de manera curva o tiene una silueta redondeada.

59. Torso sombreado: Presencia de manchas, oscurecimiento del torso o pecho.

60. Torso ancho: Ancho y desproporcionado con relación al cuerpo.

61. Cintura muy remarcada: La cintura de la figura se encuentra excesivamente delineada, reforzada a manera de cinturón o correa, destacando con relación al resto del dibujo, formando un relieve en el papel.

62. Cintura chica o estrecha: La cintura se achica en comparación al tronco hasta hacerse muy estrecha. 
63. Caderas o nalgas anchas: Caderas o nalgas son dibujadas desproporcionadas con relación al cuerpo.

64. Pelvis sombreada o borroneada: Sombreado o borrones notorios en esta parte de la figura. Un leve sombreado o borrón no se califican.

65. Bragueta o pliegues en zona pélvica: Dibujo de la bragueta o de pliegues en la zona genital.

66. Brazos omitidos: Los brazos de la figura se encuentran ausentes o interrumpidos. Los brazos detrás del cuerpo no se puntúan.

67. Brazos pegados al cuerpo: Ambos brazos de la figura se encuentran pegados al cuerpo, sin espacio visible entre el torso y los brazos.

68. Brazos levantados: Se puntúa si ambos brazos de la figura se encuentran levantados en posición horizontal o extendidos por encima de la cabeza.

69. Manos omitidas: Una o ambas manos están omitidas o incompletas (las manos escondidas detrás del cuerpo o en los bolsillos no se califican).

70. Manos ocultas: Las manos se encuentran dentro de los bolsillos o tras la espalda.

71. Manos sombreadas: Las manos están pintadas o sombreadas.

72. Manos tipo puño: Una o ambas manos están apretadas o cerradas en forma de puño.

73. Manos tipo garra: Una o ambas manos están como garra o tenaza o deformadas. Se puntúa si uno de los dedos está claramente apuntando como un clavo o cuchillo o las uñas están muy afiladas.

74. Manos muy grandes: Las manos están desproporcionadas con relación a los brazos o el resto del cuerpo.

75. Dedos omitidos: No existe esbozo o trazo de los dedos. Si la perspectiva del dibujo de la mano está de perfil, ya no se califica este ítem. Si se califica Mano Tipo Puño (ítem 72) o Garra (ítem 73), este ítem no se puntúa.

76. Más de cinco dedos en ambas manos: Si hay más de cinco dedos en ambas manos. 
77. Piernas omitidas: Una o ambas piernas están ausentes. Si se califica este ítem, ya no se puntúa Pies Omitidos (ítem 80).

78. Piernas juntas: Las piernas sólo están separadas por una línea o aparecen pegadas entre sí.

79. Piernas sombreadas: Manchas, sombras, pecas o pelos abundantes en las piernas que las hacen verse como sombreadas.

80. Pies omitidos: Uno o ambos pies de la figura están ausentes.

81. Pies desnudos: No existe ninguna indicación de calzado ni medias en una figura vestida.

82. Pies deformes: Muñones o pies semejantes a patas de animal. Esbozos de pies no se consideran como deformes.

83. Tacos: Los tacos en ambos zapatos de la figura resaltan de manera llamativa (grandes, reforzados, acentuados o sombreados).

84. Uñas: Éstas deben estar enfatizadas, remarcadas o muy visibles con respecto a la mano o los pies. Si se califica Mano Tipo Garra (ítem 73), ya no se puntúa este ítem.

85. Articulaciones remarcadas: Se califica cuando los codos o las rodillas están visiblemente delineados. Un hoyo o pequeñas rayas no se puntúan.

86. Transparencias: La figura o partes del cuerpo de la figura se translucen con respecto a la vestimenta.

87. Objeto en la boca: La figura presenta un objeto en la boca (cigarro, pipa, palillo de dientes, etc.).

88. Objeto apegado, cargado, sostenido por la figura: Presencia de maletín, sombrilla, bultos, paquetes, ramos de flores o similares.

89. Presencia de otros objetos, fondo cubierto: Presencia de árboles, casa, animales, ambientes exteriores.

90. Múltiples figuras: Más de una figura dibujada espontáneamente.

91. Marcos: Reborde o recuadro que encierra parcial o totalmente la figura.

92. Sombreado externo: Sombreado difuso en el exterior.

93. Figura sobrecargada: La figura presenta un exceso de adornos: collares, joyas, maquillaje, diseño del vestido (por ejemplo, vestimenta de dandy), grecas, sombreros, etc. 
94. Corbata: Una corbata larga y completa es dibujada.

95. Botones y/o bolsillos en exceso: La vestimenta de la figura presenta más de tres botones y/o más de un bolsillo.

96. Roturas o parches en la ropa: Parches, costuras, desgarramientos en la ropa que la hacen verse como vieja o maltratada.

97. Letras, números: Escritura o logos en la vestimenta de la figura o fuera de ella.

98. Símbolos agresivos y/o palabras obscenas: Dibujados en la ropa o en el entorno de la figura.

99. Signos bizarros: Figuras incongruentes, órganos internos o huesos, confusión del perfil y la cara, garabatos.

100. Figura uniformada: Cualquier tipo de uniforme (soldado, vaquero, policía, etc.).

101. Figura semidesnuda: El tronco de la figura está desnudo, sin camiseta, o la figura se encuentra en ropa interior o ropa de baño.

102. Figura desnuda: Completamente desnuda. Este ítem incluye la presencia o no de genitales, pero pies descalzos, pantalones cortos o camisa de manga corta no se califica. Si se califica este ítem, ya no se califica Pies Desnudos (ítem 81) ni Presencia de Genitales (ítem 103).

103. Presencia de genitales: Presencia de genitales en figura vestida. Si se puntúa Figura Desnuda (ítem102), ya no se califica este ítem.

104. Figura vacía: Como fantasma, sin rostro ni señales de ropa.

105. El primer dibujo no concuerda con el sexo del evaluado: Cuando el primer dibujo que realiza el examinado no es de su mismo sexo.

106. Figura indiferenciada sexualmente: No se puede diferenciar el género o sexo de la figura. Si se califica Figura Vacía (ítem 104) ya no se puntúa este ítem.

107. Figuras con caracteres sexuales secundarios acentuados: Se presta bastante atención en sobresaltar o acentuar los rasgos masculinos/femeninos de las figuras. Por ejemplo, mujeres con senos protuberantes, hombres musculosos, etc. 
108. Impresión femenina/masculina de la figura que no corresponde a su sexo: La figura impresiona o tiene una apariencia marcadamente femenina/masculina que no corresponde a su género dibujado.

109. Movimiento: La figura claramente evidencia la realización de una acción como caminar, correr, saltar o está ejecutando alguna actividad.

110. Figura rígida: La figura está totalmente tiesa, congelada, estática, o como en exhibición (tipo maniquí).

111. Ausencia de tono vital: La figura impresiona como falta de energía, desvitalizada, muerta.

112. Expresión hostil: La figura presenta una mirada agresiva, hostil, burlona, desafiante (ceja levantada, ceño fruncido).

113. Impacto visual desagradable: Figura percibida por el evaluador como monstruosa, grotesca. Si se puntúa Figura Vacía (ítem 104) ya no se califica este ítem, pero la puntuación del ítem es independiente de Signos Bizarros (ítem 99). 


\section{Dibujos de algunos partcipantes}

Dibujo

Hombre

Dibujo

Mujer

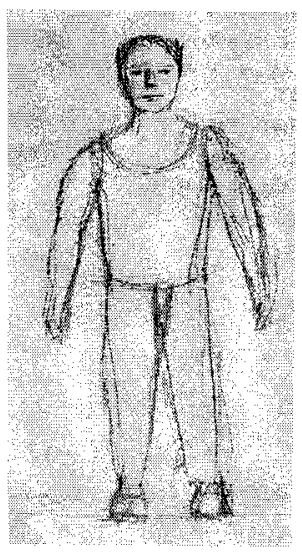

Figura 1.

Hombre homosexual, 26 años.

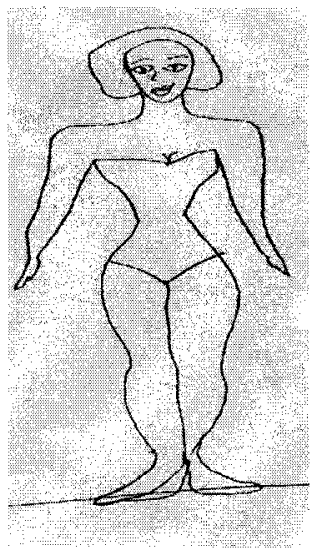

Figura 3.

Hombre homosexual, 26 años.

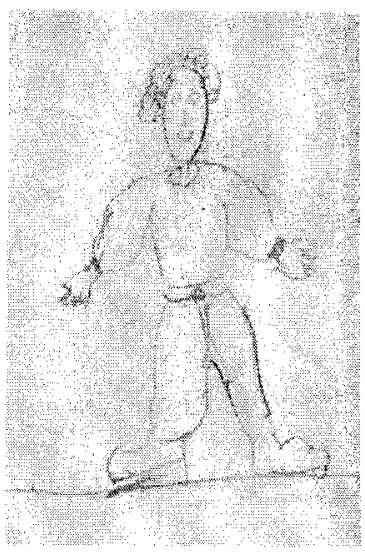

Figura 2.

Hombre heterosexual, 34 años.

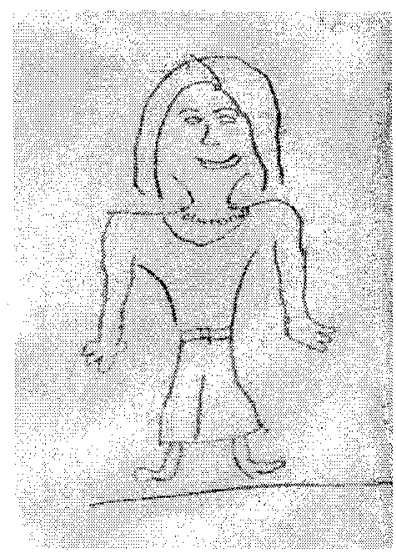

Figura 4.

Hombre heterosexual, 50 años. 
Indicadores de homosexualidad en el Dibujo de la Figura Humana

Dibujo

Sí Mismo

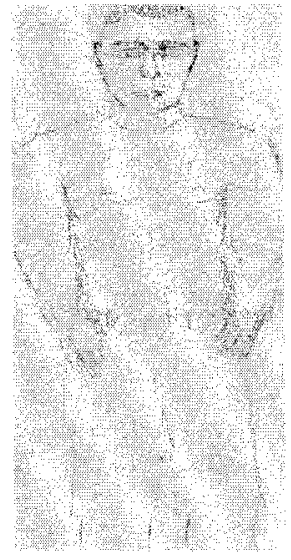

Figura 5.

Hombre homosexual, 40 años.

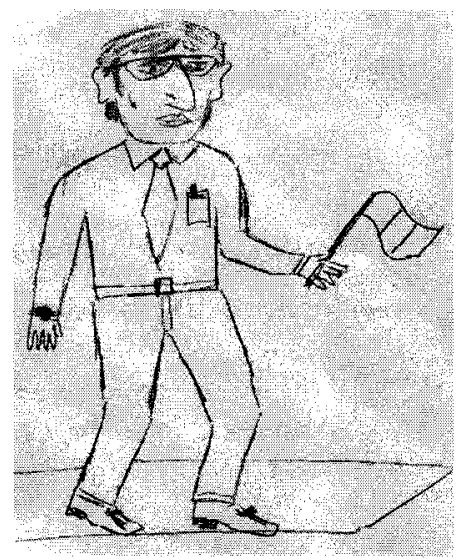

Figura 6.

Hombre heterosexual, 41 años. 